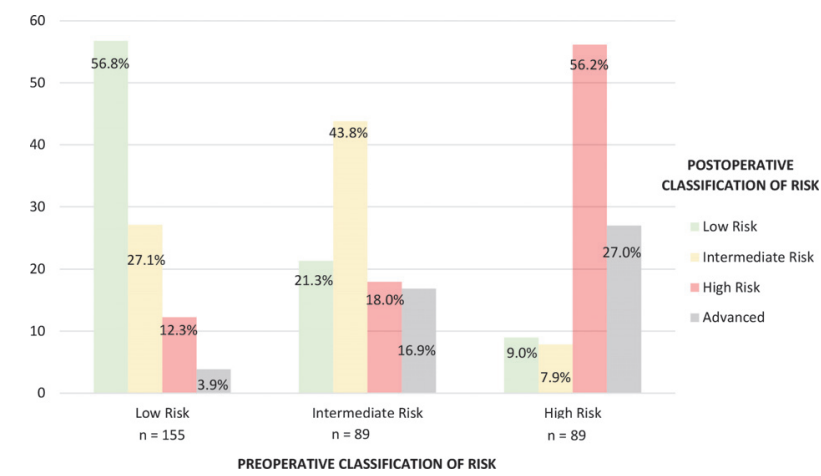

Abstract 80 Figure 1 Concordance between preoperative risk classification assessed by endometrial biopsy and magnetic resonance imaging with the final histological analysis of the surgical specimen

wrongly classified, sentinel LN procedure instead of no LN dissection could be an option offered to preoperative low risk patients to decrease the indication of second surgery for restaging and/or to avoid toxicity of adjuvant radiotherapy.

\section{TIME COURSE OF ADVERSE EVENTS DURING DOSTARLIMAB TREATMENT IN PATIENTS WITH RECURRENT OR ADVANCED ENDOMETRIAL CANCER IN THE GARNET TRIAL}

${ }^{1}$ A Oaknin*, ${ }^{2} \mathrm{~L}$ Gilbert, ${ }^{3} \mathrm{~A}$ Tinker, ${ }^{4} \mathrm{~W}$ Guo, ${ }^{4} \mathrm{E}$ Im, ${ }^{5} \mathrm{~B}$ Pothuri. ${ }^{1}$ Vall $d^{\prime}$ Hebron University Hospital, Vall d'Hebron Institute of Oncology (VHIO), Barcelona, Spain; ${ }^{2}$ McGill University Health Centre Research Institute, Montreal, Quebec, Canada; ${ }^{3} B C$ Cancer, Vancouver, British Columbia, Canada; ${ }^{4}$ GlaxoSmithKline, Waltham, MA, USA; ${ }^{5}$ Gynecologic Oncology Group (GOG) and Department of Obstetrics/Gynecology, Laura and Isaac Perlmutter Cancer Center, NYU Langone Health, New York, NY, USA

\subsection{6/ijgc-2021-ESG0.113}

Introduction/Background* Dostarlimab is a humanized programmed death-1 (PD-1) receptor monoclonal antibody that blocks interaction with the PD-1 ligands. GARNET (NCT02715284) is a phase 1 study assessing antitumor activity and safety of dostarlimab monotherapy in patients with solid tumors. Dostarlimab has shown antitumor activity in patients with mismatch repair deficient (dMMR) and mismatch repair proficient (MMRp) advanced and recurrent endometrial cancer (EC). Here we report on the time of onset of treatment-related adverse events (TRAEs) and immune-related (ir) TRAEs over the course of dostarlimab treatment in patients with dMMR (cohort A1) and MMRp (cohort A2) EC in the GARNET trial.

Methodology Patients with advanced or recurrent dMMR or MMRp EC that progressed on or after a platinum regimen received $500 \mathrm{mg}$ of dostarlimab every 3 weeks for 4 cycles, then $1000 \mathrm{mg}$ every 6 weeks (Q6W) until disease progression or discontinuation.

Result(s)* A total of 126 patients with dMMR EC and 145 patients with MMRp EC were included in the safety population. Few TRAEs were seen in $\geq 10 \%$ of patients: fatigue (17.3\%), diarrhea (14.4\%), nausea (13.7\%), and asthenia (11.1\%). The majority of cases occurred during cycles 1-3, with a peak occurrence at cycle 1 for all 4 TRAEs. Hypothyroidism was the only irTRAE seen in $\geq 5 \%$ of patients, and $94 \%$ of cases occurred between cycles 2 and 8 , with a peak

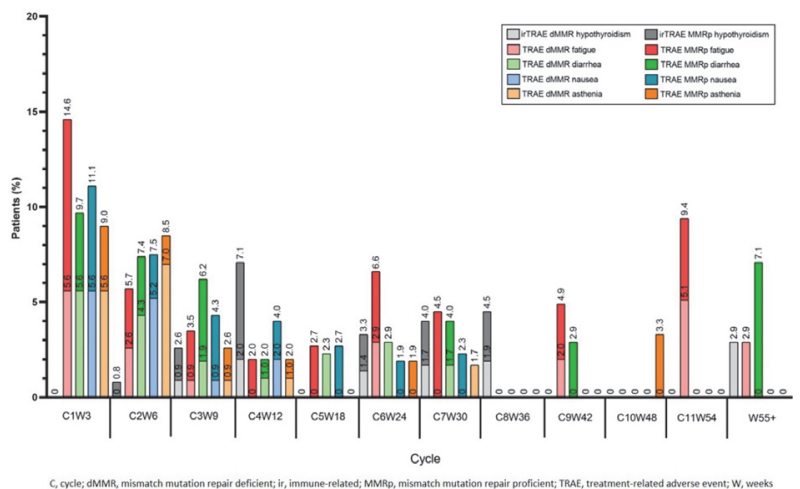

Abstract 83 Figure 1 Incidence of the most common irTRAEs and TRAEs in patients with dMMR and MMRp EC by cycle

occurrence seen at cycle 4 . irTRAEs that were seen in $\geq 1 \%$ of patients included diarrhea (4.1\%), amylase increased (2.2\%), aspartate aminotransferase increased (2.2\%), alanine aminotransferase increased $(1.8 \%)$, colitis (1.5\%), hyperglycemia (1.5\%), lipase increased (1.5\%), adrenal insufficiency (1.1\%), and hyperthyroidism (1.1\%).

Conclusion* When analyzed over the dMMR and MMRp EC safety population of the GARNET trial, dostarlimab has an acceptable safety profile with manageable adverse events. irTRAEs and TRAEs were seen in a low percentage of patients and were seen more frequently earlier in the time course of dostarlimab treatment. No increase in the rate of TRAEs or irTRAEs was seen when changing to the 1000-mg Q6W dose.

C, cycle; dMMR, mismatch mutation repair deficient; ir, immune-related; MMRp, mismatch mutation repair proficient; TRAE, treatment-related adverse event; W, weeks

\section{ENDOMETRIAL CANCER IMMUNOHISTOCHEMICAL RISK STRATIFICATION IN A LARGE UTERINE-CONFINED CANCER SERIES}

${ }^{1}$ E Perrone*, ${ }^{2} \mathrm{~F}$ De Felice, ${ }^{1} \mathrm{I}$ Capasso, ${ }^{3} \mathrm{D}$ Arciuolo, ${ }^{1} \mathrm{E}$ Distefano, ${ }^{1} \mathrm{D}$ Lorusso, ${ }^{3} \mathrm{GF}$ Zannoni, ${ }^{1} \mathrm{G}$ Scambia, ${ }^{1} \mathrm{~F}$ Fanfani. ${ }^{1}$ Agostino Gemelli University Polidinic, UOC Ginecologia Oncologica, Dipartimento per la salute della Donna e del Bambino e della Salute Pubblica, Roma, Italy; ${ }^{2}$ Policlinico Umberto I, Radiotherapy, Roma, Italy; ${ }^{3}$ Agostino Gemelli University Policlinic, Gyneco-pathology and Breast Pathology Unit, Roma, Italy

\subsection{6/ijgc-2021-ESG0.114}

Introduction/Background* Nowadays, after the recent insights about the molecular Endometrial cancer (EC) classification, the usual key histological parameters (i.e histotype and grade) have been shown to have poor reproducibility and adequacy in EC stratification risk. The need to define a more precise guidance of surgical and adjuvant approaches has suggested the possibility to refine the prognostic assessing, considering other EC characteristics. Inspired by these concepts, the aim of this study is to assess the clinical reproducibility and the oncological validity of the EC risk stratification based on the molecular information given by the immunohistochemistry (IHC). Methodology Retrospective IHC analyses were conducted in a large series of 778 pre-operative uterine-confined ECs, studying the presence/absence of MLH1, MSH2, MSH6, to define the mismatch repair (MMR) stable or instable phenotype; the 
Abstract 86 Table 1 Distribution of clinicopathological parameters

\begin{tabular}{|c|c|c|c|c|c|}
\hline & MMRs & MMRi & p53+ & Total & p-value \\
\hline $\begin{array}{l}\text { Number of } \\
\text { patients }\end{array}$ & 480 & 170 & 128 & 778 & \\
\hline Age at diagnosis & & & & & $<0.001$ \\
\hline Mean (SD) & $61.4(11.1)$ & $63.8(10.5)$ & $68.8(9.4)$ & $63.1(11.0)$ & \\
\hline Range & $25.0-87.0$ & $29.0-88.0$ & $46.0-89.0$ & $25.0-89.0$ & \\
\hline BMI & & & & & 0.136 \\
\hline Mean (SD) & $30.4(8.9)$ & $30.9(8.1)$ & $29.0(6.7)$ & $30.3(8.4)$ & \\
\hline Range & $16.8-121.0$ & $17.4-64.1$ & $17.2-51.9$ & $16.8-121.0$ & \\
\hline FIGO_stage & & & & & $<0.001$ \\
\hline IA & $298(62.1 \%)$ & $86(50.6 \%)$ & $49(38.3 \%)$ & $433(55.7 \%)$ & \\
\hline IB & $113(23.5 \%)$ & $44(25.9 \%)$ & $37(28.9 \%)$ & $194(24.9 \%)$ & \\
\hline ॥ & $28(5.8 \%)$ & $16(9.4 \%)$ & $19(14.8 \%)$ & $63(8.1 \%)$ & \\
\hline IIIA & $5(1.0 \%)$ & $3(1.8 \%)$ & $3(2.3 \%)$ & $11(1.4 \%)$ & \\
\hline IIIB & $5(1.0 \%)$ & $0(0.0 \%)$ & $3(2.3 \%)$ & $8(1.0 \%)$ & \\
\hline IIIC1 & $25(5.2 \%)$ & $14(8.2 \%)$ & $12(9.4 \%)$ & $51(6.6 \%)$ & \\
\hline IIIC2 & $1(0.2 \%)$ & $2(1.2 \%)$ & $0(0.0 \%)$ & $3(0.4 \%)$ & \\
\hline IVA & $0(0.0 \%)$ & $3(1.8 \%)$ & $1(0.8 \%)$ & $4(0.5 \%)$ & \\
\hline IVB & $5(1.0 \%)$ & $2(1.2 \%)$ & $4(3.1 \%)$ & $11(1.4 \%)$ & \\
\hline Early stage (I-II) & & & & & 0.005 \\
\hline no & $41(8.5 \%)$ & $24(14.1 \%)$ & $23(18.0 \%)$ & $88(11.3 \%)$ & \\
\hline yes & $439(91.5 \%)$ & $146(85.9 \%)$ & $105(82.0 \%)$ & $690(88.7 \%)$ & \\
\hline Tumor grade & & & & & $<0.001$ \\
\hline G1-2 & $420(87.5 \%)$ & $131(77.1 \%)$ & $6(4.7 \%)$ & $557(71.6 \%)$ & \\
\hline G3 & $60(12.5 \%)$ & $39(22.9 \%)$ & $122(95.3 \%)$ & $221(28.4 \%)$ & \\
\hline Histotype & & & & & $<0.001$ \\
\hline Type I & $450(93.8 \%)$ & $161(94.7 \%)$ & $16(12.5 \%)$ & $627(80.6 \%)$ & \\
\hline Type II & $30(6.2 \%)$ & $9(5.3 \%)$ & $112(87.5 \%)$ & $151(19.4 \%)$ & \\
\hline LVSI & & & & & $<0.001$ \\
\hline Negative & $401(83.7 \%)$ & $128(76.2 \%)$ & $76(61.3 \%)$ & $605(78.5 \%)$ & \\
\hline Positive & $78(16.3 \%)$ & $40(23.8 \%)$ & $48(38.7 \%)$ & $166(21.5 \%)$ & \\
\hline $\begin{array}{l}\text { Myometrial } \\
\text { invasion }\end{array}$ & & & & & 0.003 \\
\hline$\leq 50$ & $324(67.6 \%)$ & $101(59.8 \%)$ & $67(52.3 \%)$ & $492(63.4 \%)$ & \\
\hline$>50$ & $155(32.4 \%)$ & $68(40.2 \%)$ & $61(47.7 \%)$ & $284(36.6 \%)$ & \\
\hline $\begin{array}{l}\text { Tumor dimension } \\
\text { (class) }\end{array}$ & & & & & 0.584 \\
\hline$\leq 20 \mathrm{~mm}$ & $117(24.4 \%)$ & 37 (21.8\%) & $27(21.1 \%)$ & $181(23.3 \%)$ & \\
\hline$>20 \mathrm{~mm}$ & $360(75.0 \%)$ & $133(78.2 \%)$ & 101 (78.9\%) & $594(76.3 \%)$ & \\
\hline Tumor dimension & & & & & $<0.001$ \\
\hline Mean (SD) & $33.1(18.1)$ & $\begin{array}{l}35.200 \\
(16.314)\end{array}$ & $40.3(25.9)$ & 34.8 (19.4) & \\
\hline Range & $1.0-140.0$ & $1.0-95.0$ & $1.0-150.0$ & $1.0-150.0$ & \\
\hline Pathological & & & & & 0.057 \\
\hline lymph node status & & & & & \\
\hline Negative & $468(97.5 \%)$ & $159(93.5 \%)$ & $123(96.1 \%)$ & $750(96.4 \%)$ & \\
\hline Positive & $12(2.5 \%)$ & $11(6.5 \%)$ & $5(3.9 \%)$ & $28(3.6 \%)$ & \\
\hline $\begin{array}{l}\text { Post-surgical } \\
\text { treatment }\end{array}$ & & & & & 0.082 \\
\hline None & $212(45.0 \%)$ & $90(54.9 \%)$ & $55(45.1 \%)$ & $357(47.2 \%)$ & \\
\hline Any & $259(55.0 \%)$ & $74(45.1 \%)$ & $67(54.9 \%)$ & $400(52.8 \%)$ & \\
\hline Adjuvant CHT & & & & & 0.093 \\
\hline No & $282(59.9 \%)$ & $113(68.1 \%)$ & $69(56.6 \%)$ & $464(61.1 \%)$ & \\
\hline Yes & $189(40.1 \%)$ & $53(31.9 \%)$ & $53(43.4 \%)$ & 295 (38.9\%) & \\
\hline Adjuvant RT & & & & & 0.540 \\
\hline No & $229(47.7 \%)$ & $89(52.4 \%)$ & $65(50.8 \%)$ & $383(49.2 \%)$ & \\
\hline Yes & 251 (52.3\%) & $81(47.6 \%)$ & $63(49.2 \%)$ & $395(50.8 \%)$ & \\
\hline Risk class 2020 & & & & & $<0.001$ \\
\hline Low & $260(54.2 \%)$ & $72(42.4 \%)$ & $1(0.8 \%)$ & $333(42.8 \%)$ & \\
\hline Intermediate & $79(16.5 \%)$ & $25(14.7 \%)$ & $11(8.6 \%)$ & $115(14.8 \%)$ & \\
\hline
\end{tabular}

\begin{tabular}{lllll} 
High/ & $80(16.7 \%)$ & $44(25.9 \%)$ & $8(6.2 \%)$ & $132(17.0 \%)$ \\
intermediate & & & & \\
High & $56(11.7 \%)$ & $27(15.9 \%)$ & $104(81.2 \%)$ & $187(24.0 \%)$ \\
Advanced/ & $5(1.0 \%)$ & $2(1.2 \%)$ & $4(3.1 \%)$ & $11(1.4 \%)$ \\
Metastatic & & & & \\
\hline
\end{tabular}
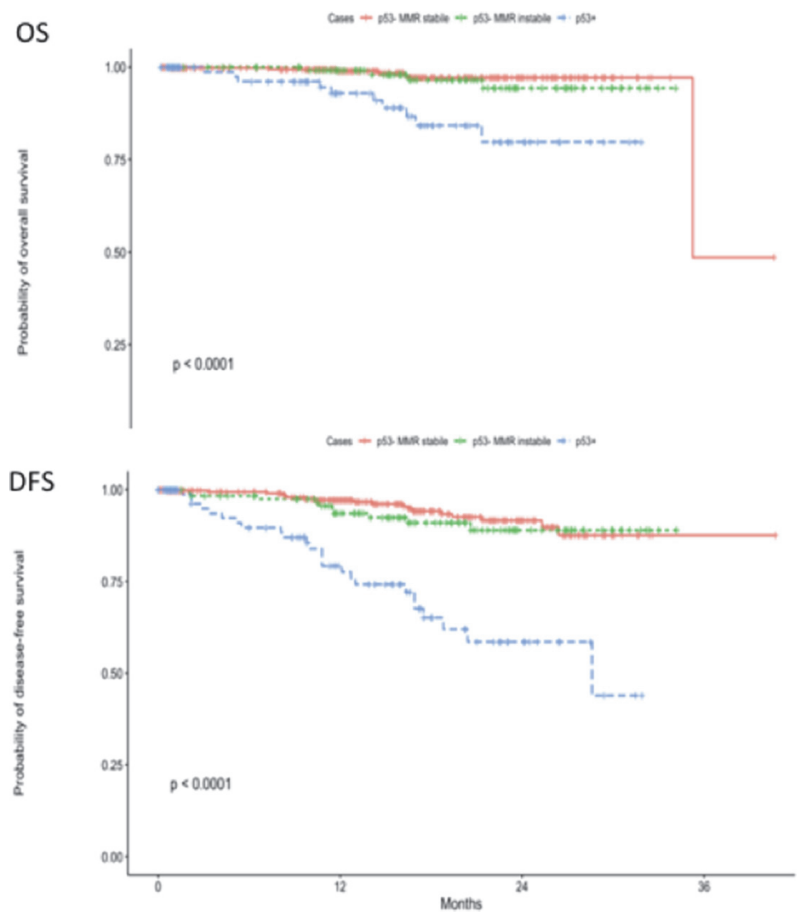

\section{Abstract 86 Figure 1}

presence of p53 mutations and other molecular features. The molecular profile was correlated with histological, clinical and prognostic EC patients data.

Result(s)* Based on the IHC, we defined 3 EC populations: MMR stable (MMRs), instable (MMRi) and p53 mutated $(\mathrm{p} 53+)$ patients. Our result demonstrated that the IHC stratification statistically correlated with the most relevant anatomoclinical features: FIGO stage $(p<0.001)$, grading $(12,5 \%$ G3 in MMRs vs $22.9 \%$ in MMRi vs $95.3 \%$ in $\mathrm{p} 53+, \mathrm{p}<0.001$ ), histotype (Type II $6.2 \%$ in MMRs vs $5.3 \%$ in MMRi vs $87.5 \%$ in $\mathrm{p} 53+, \mathrm{p}<0.001$ ), presence of LVSI (positive in $16.3 \%$ in MMRs vs $23.8 \%$ in MMRi vs $38.7 \%$ in $\mathrm{p} 53+$, $\mathrm{p}<0.001)$, myometrial invasion and tumor dimension $(\mathrm{p}=0.003$ and $\mathrm{p}<0.001$ respectively). Again, the 3 IHC populations statistically reflected the EC risk class ESGO-ESMO-ESP classification $2020(p<0.001) \quad$ (table 1$)$. These results were confirmed also in Kaplan-Meier curves in terms of over-all survival (OS) and disease-free survival (DFS) $(\mathrm{p}<0.0001) \quad$ (figure 1). Moreover, the multivariate analyses demonstrated that absence of estrogen receptor (ER) impacted the OS $(p=0.011)$ and, the Age $>60$ years and the ER-status in DFS $(p=0.004$ and $\mathrm{p}=0.041$ ).

Conclusion* In this large series, we demonstrated that the pragmatic and sistematic use of IHC may have an important role to properly stratify, in terms of histological features and clinical outcome, the pre-operative uterine-confined EC patients. 